\title{
SUR CERTAINES VARIÉTÉS HOMOGÈNES COMPLEXES
}

\author{
YOZÔ MATSUSHIMA
}

Introduction. Soit $V$ une variété complexe satisfaisant aux conditions suivantes :

1) un groupe de Lie semi-simple connexe $G$ opère sur $V$ de manière transitive et holomorphe;

2) la varieté $V$ admet une mesure invariante par $G^{11}$.

On dira par abus de langage que la variété $V$ est semi-simple. On se propose d'étudier dans ce travail des variétés complexes compactes semi-simples. Les variétés de ce genre font l'objet de deux travaux de Wang [5], [6]. Dans le mémoire [5] il a étudié le cas simplement connexe et dans [6] le cas parallélisable. Le résultat obtenu dans ce travail montre que toute variété complexe compacte semi-simple est en un certain sens une combinaison de deux types de variétés étudiées par Wang. On établit en effet le théorème suivant.

THÉoRÈme 1. Soit $V$ une variété complexe compacte semi-simple. Alors $V$ est un espace fibré holomorphe de base $W$ et de fibre $F$. La base $W$ est un espace homogène kählérien d'un groupe de Lie compact semi-simple connexe et la fibre $F$ est un espace quotient d'un groupe de Lie complexe réductif connexe par un sous-groupe discret.

Ce théorème généralise un théorème bien connu de Wang [5]. Voir également un mémoire récent de Hano et Kobayashi [3]. L'énoncé détaillé du théorème 1 sera donné au paragraphe 4 sous la forme du théorème $1^{\prime}$. On trouvera aussi une méthode pour construire les variétés complexes compactes semi-simples.

On déduira du théorème 1 et d'un théorème de Wang [6] le théorème suivant.

Received October 15, 1960.

1) Pour qu'il en soit ainsi il faut et il suffit que le groupe d'isotropie de $G$ en un point de $V$ soit unimodulaire. 
THÉoRème 2. Soit $V$ une variété kählérienne compacte semi-simple. Alors $V$ est un espace homogène kählérien d'un groupe de Lie compact semi-simple connexe.

Ainsi dans le cas d'une variété kählérienne compacte l'existence d'une mesure invariante entraine l'existence d'une métrique kählérienne invariante.

1. Soit $G$ un groupe de Lie semi-simple connexe. Soit a l'algèbre de Lie du groupe $G$ et soit $\mathfrak{g}=a_{1}+\cdots+a_{k}$, où $a_{i}(i=1, \ldots, k)$ sont des idéaux simples de я. Soient $A_{i}(i=1, \ldots, k)$ les sous-groupes invariants de Lie de $G$ correspondant à $a_{i}$. Supposons que les sous-groupes $A_{i}(i=1, \ldots, s)$ soient compacts et que les autres $A_{j}(j=s+1, \ldots, k)$ ne soient pas compacts. Soient $G_{1}=A_{1} \ldots A_{s}$ et $G_{2}=A_{s+1} \ldots A_{k}$. Alors $G=G_{1} \cdot G_{2}=G_{2} \cdot G_{1}$. On appellera $G_{1}$ la composante compacte de $G$ et $G_{2}$ la composante ouverte de $G$.

A. Rappelons d'abord un résultat récent de Borel [1]. Soit $G$ un groupe de Lie. On dira qu'un sous-groupe $B$ de $G$ possède la propriété $(S)$ dans $G$ si pour tout voisinage $U$ de l'élément neutre dans $G$ et pour tout élément $g$ de $G$ il existe un entier $n>0$ tel que $g^{n} \in U \cdot B \cdot U$. Soient $G$ et $G^{\prime}$ des groupes de Lie connexes et soit $\varphi$ un homomorphisme de $G$ sur $G^{\prime}$. Soit $B$ ùn sous-groupe de $G$ possèdant la propriété $(S)$ dans $G$. Alors l'image $\varphi(B)$ de $B$ dans $G^{\prime}$ possède aussi la propriété $(S)$ dans $G^{\prime}$. D'aprés un lemme de Selberg, si l'espace quotient $G / B$ possède une mesure invariante dont la mesure totale est finie, le sous-groupe $B$ possède la propriété $(S$ ) dans $G$ (voir [1]).

A. Borel a démontré le théorème suivant.

Théorème de Borel. Soit $G$ un groupe de Lie semi-simple connexe dont la composante compacte se réduit à l'élément neutre. Soit $B$ un sous-groupe possèdant la propriété (S) dans $G$. Alors toute sous-algèbre de l'algèbre de Lie g du groupe $G$ qui est stable par ad.x pour tout $x \in B$ est un idéal de g.

Utilisant ce théorème de Borel on va démontrer un lemme sur les espaces homogènes symplectiques.

Un espace homogène $V=G / B$ d'un groupe de Lie $G$ est dit homogène symplectique, s'il existe sur $V$ une 2 -forme extérieure $\psi$ partout de rang maximal et invariante par $G$ telle que $d \psi=0$.

Lemme. Soit $V=G / B$ un espace homogène symplectique d'un groupe de 
Lie semi-simple connexe $G$. Supposons que $G$ opère sur $V$ de manière presque effective et que le sous-groupe $B$ possède la propriété (S) dans $G$. Alors le groupe G est compact.

Soient $G_{1}$ et $G_{2}$ la composante compacte et la composante ouverte de $G$ respectivement. Soit $\widetilde{G}=G_{1} \times G_{2}$. Soit $\varphi$ l'homomorphisme canonique de $\widetilde{G}$ sur $G$ défini par $\varphi\left(g_{1}, g_{2}\right)=g_{1} \cdot g_{2}$. Soit $\widetilde{B}$ l'image inverse par $\varphi$ du sous-groupe $B$ de $G$. On peut identifier l'espace quotient $\tilde{G} / \widetilde{B}$ avec $G / B$ par l'application $\widetilde{g} \cdot \widetilde{B} \rightarrow \breve{c}(\widetilde{g}) \cdot B$. Alors $\widetilde{G} / \widetilde{B}$ est un espace homogène symplectique et, le noyau de l'homomorphisme $\varphi$ étant discret, $\widetilde{G}$ opère de manière presque effective sur $\widetilde{G} / \widetilde{B}$. De plus, le noyau de $\varphi$ ètant dans le centre de $\hat{G}$, le sous-groupe $\widetilde{B}$ possède la propriété $(S)$ dans $\tilde{G}$ (cf. [1]). Il suffit donc de démontrer le lemme pour l'espace homogène $\widetilde{G} / \widetilde{B}$. Pour simplifier les notations on supposera que $\widetilde{G}=G$, c'est-à-dire que $G=G_{1} \times G_{2}$. On désignera les algèbres de Lie des groupes $G, G_{1}, G_{2}$ par $\mathfrak{g}, \mathfrak{g}_{1}, \mathfrak{g}_{2}$. Alors $\mathfrak{g}=\mathfrak{g}_{1}+\mathfrak{g}_{2}$. Soit $\pi_{2}$ la projection canonique de $G=G_{1} \times G_{2}$ sur $G_{2}$. On désignera par la même $\pi_{2}$ la projection canonique de $g$ sur $g_{2}$. Le sous-groupe $B$ possède la propriété $(S)$ dans $G$ et par suite l'image $\pi_{2}(B)$ de $B$ possède la même propriété dans $G_{2}$. Or la composante compacte du groupe semi-simple $G_{2}$ se réduit à l'élément neutre. Alors il résulte du théorème de Borel que l'algèbre $\pi_{2}(\mathfrak{b})$ du groupe $\pi_{2}(B)$ est un idéal de $g_{2}$. D'autre part, la variété $G / B$ étant un espace homogène symplectique du groupe semi-simple $G$, l'algèbre b du groupe d'isotropie $B$ est le centralisateur dans $g$ d'un élément $W$ de $g$ (voir [2], [4]). Soit $W=W_{1}+W_{2}, W_{1} \in g_{1}, W_{2} \in$ $\mathfrak{g}_{2}$. Soit $\mathfrak{b}_{i}$ le centralisateur de $W_{i}$ dans $\mathfrak{g}_{i}(i=1,2)$. Alors $\mathfrak{b}=\mathfrak{b}_{1}+\mathfrak{b}_{2}, \mathfrak{b}_{i}=\mathfrak{b} \cap \mathfrak{g}_{i}$. Par suite on a $\pi_{2}(\mathfrak{b})=\mathfrak{b}_{2}$ et $\mathfrak{b}_{2}$ est un idéal de $\mathfrak{g}_{2}$ et donc de $\mathfrak{g}$. Or on a supposé que $G$ opère de manière presque effective sur $G / B$. Alors l'algèbre $\mathfrak{b}$ ne contient pas d'idéal de $g$ de dimension positive. Par conséquent, on a $\mathfrak{b}_{2}=(0)$ et $W_{2}=0$, car $W_{2}$ est un élément de $\mathfrak{b}_{2}$. Alors $W=W_{1} \in \mathfrak{g}_{1}$ et le centralisateur $\mathfrak{b}$ de $W$ dans $g$ contient l'idéal $g_{2}$. Alors $g_{2}=(0)$ et par suite $G=G_{1}$. Le groupe $G$ est ainsi compact.

B. Variétés complexes parallélisables (voir Wang [6]). Soit $V$ une variété complexe compacte. Soit a l'algèbre de Lie des champs de vecteurs analytiques sur $V$. On sait que a est de dimension finie et engendre le plus grand groupe connexe d'automorphismes de $V$. Soit $J$ le champ de tenseurs définissant la 
structure complexe de $V$. Si $X \in \mathfrak{a}$, on a $J \cdot X \in \mathfrak{a}$ et $J \cdot[X, Y]=[J \cdot X, Y]=$ $[X, J \cdot Y]$ quels que soient $X, Y \in a$. Le tenseur $J$ définit donc une structure d'algèbre de Lie complexe de $a$. Une variété complexe compacte $V$ sera dite parallélisable s'il existe des champs de vecteurs $X_{1}, \ldots, X_{n}$ dans a ( $n$ étant la dimension complexe de $V$ ) qui sont linéairement indépendants sur les nombres complexes en chaque point de $V$. Cette dernière condition signifie que, si $\sum_{k=1}^{n}\left[a_{k} \cdot\left(X_{k}\right)_{p}+b_{k} \cdot J\left(X_{k}\right)_{p}\right]=\ell$ en un point $p \in V\left(a_{k}, b_{k}\right.$ étant des nombres réels), on a $a_{1}=\cdots a_{n}=0, b_{1}=\cdots=b_{n}=0$. On voit alors que $X_{1}, \ldots, X_{n}$ forment une base complexe de l'algèbre complexe $a$. Il résulte de cette définition que, si $V$ est parallélisable et connexe, le plus grand groupe connexe d'automorphismes $A_{0}$ de $V$ est transitif sur $V$ et que le groupe d'isotropie de $A_{0}$ en un point de $V$ est discret.

Soit maintemant $L$ un groupe de Lie connexe opérant de manière transitive, holomorphe et presque effective sur $V$ parallélisable. Soit $\mathfrak{l}$ l'algèbre des champs de vecteurs sur $V$ définis par les transformations de groupes à un paramètre dans $L$. Alors $\mathfrak{l}$ est une sous-algèbre de $a$ et isomorphe à l'algèbre du groupe $L$. Le groupe $L$ étant transitif sur $V$, la dimension réelle de $\mathfrak{l}$ est plus grande ou égale à celle de $V$. Or la dimension complexe de $V$ est égale à celle de $a$. On a alors $\mathfrak{I}=a$. Le groupe $L$ est alors un groupe de Lie complexe et le groupe d'isotropie de $L$ en un point de $V$ est discret.

2. Démonstration du théorème 1. Soit $V$ une variété complexe compacte semi-simple. Soit $G$ un groupe de Lie semi-simple connexe opérant sur $V$ de manière transitive, holomorphe et presque effective. On suppose que $V$ possède une mesure invariante par $G$. La variété $V$ étant compacte, la mesure totale de $V$ est finie. Soit $V=G / B$. Alors le sous-groupe $B$ possède la propriété $(S)$ dans $G$ (voir [1]). Soient $G_{1}$ et $G_{2}$ la composante compacte et la composante ouverte de $G$ respectivement. On peut supposer sans gêner la généralité que $G=G_{1} \times G_{2}$. D'après un résultat récent de Hano-Kobayashi [3], il existe un sous-groupe fermé $L$ de $G$ contenant $B$ et possédant les deux propriétés suivantes :

A) l'espace quotient $G / L$ est homogène symplectique;

B) l'espace quotient $L / B$ est connexe et une sous-variété complexe de $G / B$. De plus, L/B est parallelisable. 
Soit maintenant $M$ le plus grand sous-groupe connexe invariant de $G$ contenu dans $L$. Alors $M$ est fermé. Puisque $L$ contient $B$ et que $B$ possède la propriété $(S)$ dans $G, L$ possède aussi la même propriéte dans $G$. Par suite le sous-groupe $L / M$ de $G / M$ possède la propriété $(S)$ dans $G / M$. Or le groupe $G / M$ opère de manière transitive et presque effective sur l'espace homogène symplectique $G / L$. Il résulte alors du lemme que le groupe $G / M$ est compact. De plus, le groupe $G / M$ étant compact semi-simple, le groupe $L / M$ est connexe et coïncide avec le centralisateur d'un tore de $G / M$ (cf. [2], [4]).

Le groupe $G / M$ étant compact, le sous-groupe connexe invariant fermé $M$ de $G$ doit être de la forme $M=M_{1} \times G_{2}$, où $M_{1}$ est un sous-groupe connexe invariant fermé du groupe compact $G_{1}$. Les groupes $M$ et $L / M$ étant connexe, le groupe $L$ l'est aussi. Puisque $L$ contient $M, L$ est de la forme $L=L_{1} \times G_{2}$, $L_{1} \supset M_{1}$, où $L_{1}$ est un sous-groupe connexe fermé du groupe compact $G_{1}$.

Or la variété $F=L / B$ est une sous-variété complexe parallélisable de $G / B$. Soit $N$ le plus grand sous-groupe connexe invariant de $L$ contenu dans $B$. Alors $N$ est fermé et le groupe quotient $L^{\prime}=L / N$ opère de manière transitive et presque effective sur $F$. Par suite le groupe $L^{\prime}$ est un groupe de Lie complexe et le groupe d'isotropie de $L^{\prime}$ en un point de $F$ est discret (cf. 1, B). Or ce groupe d'isntropie est isomorphe au groupe $B / N$. Par conséquent, $B / N$ est un sous-groupe discret du groupe $L^{\prime}=L / N$ et ceci montre que $N$ est égal à la composante connexe de l'élément neutre $B_{0}$ de $B$. On a ainsi démontré le résultat suivant: la composante connexe de l'élément neutre $B_{0}$ de $B$ est un sous-groupe invariant de $L$ et le groupe quotient $L^{\prime}=L / B_{0}$ est un groupe de Lie complexe opérant de manière transitive et presque effective sur la variété complexe compacte parallélisable $F=L / B$.

Or on a supposé que le groupe $G$ opère de manière presque effective sur $G / B$. Alors le groupe $B_{0}$ ne contient pas de sous-groupe invariant connexe de dimension positive de $G$. Le groupe $B_{0}$ étant invariant dans le groupe réductif $L=L_{1} \times G_{2}, B_{0}$ est contenu dans $L_{1}$, sinon $B_{0}$ contiendrait une composante simple de $G_{2}$ qui est un sous-groupe invariant de $G$.

Considérons maintemant l'image dans $L^{\prime}$ du sous-groupe invariant compact $L_{1}$ de $L$. L'image dans $L^{\prime}$ de la partie semi-simple de $L_{1}$ est un sous-groupe invariant compact semi-simple de $L^{\prime}$. Le groupe $L^{\prime}$ étant complexe, $L^{\prime}$ ne contient pas de sous-groupe invariant compact semi-simple de dimension positive. 
Par suite l'image dans $L^{\prime}$ de la partie semi-simple de $L_{1}$ se réduit à l'élément neutre. La partie semi-simple de $L_{1}$ est donc contenue dans $B_{0}$.

Revenons maintenant au sous-groupe invariant fermé connexe $M$ de $G$. On a $M=M_{1} \times G_{2}, M_{1} \subset L_{1}$. Le groupe $M_{1}$ étant invariant dans $G_{1}, M_{1}$ est semisimple et donc dans la partie semi-simple de $L_{1}$. Alors $M_{1}$ est contenu dans $B_{0}$ et par suite $M_{1}=(e)$, car $M_{1}$ est invariant dans $G$. On a ainsi montré que $M=G_{2}$. D'autre part, on a déjà dit que $L / M$ est le centralisateur d'un tore de $G / M$. Or $L / M \cong L_{1}$ et $G / M \cong G_{1}$ et par suite $L_{1}$ est le centralisateur d'un tore de $G_{1}$.

Résumons ce que on a démontré: $L=L_{1} \times G_{2}$ et $L_{1}$ est le centralisateur. d'un tore de $G_{1}$. La composante connexe de l'élément neutre $B_{0}$ du groupe $B$ est un sous-groupe invariant du $L_{1}$ contenant la partie semi-simple de $L_{1} . B_{0}$ est donc un $C$-sous-groupe du groupe semi-simple compact $G_{1}$ au sens de Wang [5]. Le groupe $L / B_{0}$ est complexe et opère de manière transitive et presque effective sur la variété complexe compacte parallélisable $F=L / B$.

3. Suite de la démonstration du théorème 1 . On va montrer maintenant les faits suivants :

1) la variété $L_{1} \cdot B / B$ est une sous-variété complexe de $G / B$;

2) le groupe $G_{2}$ est un groupe complexe et la variété $G_{2} \cdot B / B$ est une sousvariété complexe de $G / B$.

Démonstration de 1). Soit $\pi$ la projection canonique de $G$ sur $G / B$ et soit $\pi(e)=o, e$ étant l'élément neutre de $G$. Soit $\mathfrak{l}_{1}$ l'algèbre de Lie des champs de vecteurs analytiques sur $G / B$ définis par les transformations de groupes à un paramètre dans $L_{1}$. La variété $L_{1} \cdot B / B$ est égale à l'orbite du point $o$ par $L_{1}$ et contenue dans $F=L / B$. L'image $L_{1}^{\prime}$ de $L_{1}$ dans le groupe $L^{\prime}=L / B_{0}$ est égale au centre connexe de $L^{\prime}$. Le groupe $L^{\prime}$ étant complexe, le centre connexe $L_{1}^{\prime}$ de $L^{\prime}$ est aussi complexe. Cela signifie ceci: pour tout $X \in \mathfrak{l}_{1}$, il existe un $Y \in \mathfrak{l}_{1}$ tel que $J \cdot X_{p}=Y_{p}$ pour tout $p \in F$, où $J$ désigne le champ de tenseurs sur $G / B$ définissant la structure complexe de $G / B$ (cf. 1, B ; remarquons que, $F$ étant une sous-variété complexe de $G / B$, le tenseur sur $F$ définissant la structure complexe de $F$ est égal à la restriction de $J$ ). La variété $L_{1} \cdot B / B$ étant contenue dans $F$ et égale à l'orbite du point $o$ par $L_{1}$, il résulte de ce que on a montré que pour tout point $p$ de $L_{1} \cdot B / B$ et pour tout vecteur $X_{p}$ en 
$p$ tangent à $L_{1} \cdot B / B$, le vecteur $l \cdot X_{p}$ est tangent à $L_{1} \cdot B / B$. Ceci montre que $L_{1} \cdot B / B$ est une sous-variété complexe de $G / B$.

Démonstration de 2). On utilisera les notations introduites ci-dessus. Soit $G_{2}^{\prime}$ l'image de $G_{2}$ dans $L^{\prime}=L / B_{0}$. Alors $G_{2}^{\prime}$ est égale à la partie semi-simple du groupe complexe réductif $L^{\prime}$. Alors le groupe $G_{2}^{\prime}$ est aussi complexe. Comme on le voit facilement, $G_{2}$ est localement isomorphe à $G_{2}^{\prime}$. Par suite, le groupe $G_{2}$ est aussi un groupe complexe. Le fait que $G_{2} \cdot B / B$ est une sous-variété complexe de $G / B$ peut être démontré d'une manière analogue à celle de la démontration de 1 ).

4. Fin de la démonstration du théorème 1 . On va montrer maintenant les faits suivants :

1) la variété $G_{1} \cdot B / B$ est une sous-variété complexe de $G / B$;

2) la variété $G / L$ se munit d'une structure complexe invariante par $G$ telle que la projection canonique de $G / B$ sur $G / L$ soit holomorphe.

Le groupe $L_{1}$ étant compact, il existe un sous-espace vectoriel $\mathfrak{w}_{1}$ de l'algèbre $g_{1}$ du groupe $G_{1}$ tel que

$$
\mathfrak{g}_{1}=\mathfrak{w}_{1}+\mathfrak{l}_{1}, \quad \mathfrak{w}_{1} \cap \mathfrak{l}_{1}=(0), \quad\left[\mathfrak{l}_{1}, \mathfrak{w}_{1}\right] \subset \mathfrak{w}_{1},
$$

où $\mathfrak{l}_{1}$ désigne l'algèbre du groupe $L_{1}$. Puisque l'algèbre $\mathfrak{b}$ du groupe $B_{0}$ est un idéal de $\mathfrak{l}_{1}$ contenant la partie semi-simple de $\mathfrak{l}_{1}$, il existe un sous-espace vectoriel $\mathfrak{n}_{1}$ dans le centre de $\mathfrak{l}_{1}$ tel que

$$
\mathfrak{l}_{1}=\mathfrak{n}_{1}+\mathfrak{b}, \quad \mathfrak{n}_{1} \cap \mathfrak{b}=(0), \quad\left[\mathfrak{n}_{1}, \mathfrak{b}\right]=(0) .
$$

De (1) et (2) résulte

$$
g_{1}=m_{1}+\mathfrak{b}, \quad m_{1}=n_{1}+\mathfrak{w}_{1}, \quad\left[\mathfrak{b}, m_{1}\right] \subset m_{1} .
$$

Or on a $g=g_{1}+g_{2} \quad$ Alors de (3) résulte

$$
g=m+\mathfrak{b}, \quad m=m_{1}+g_{2}, \quad[\mathfrak{b}, m]=\left[\mathfrak{b}, m_{1}\right] \subset m_{1} .
$$

On désignera par $X_{\mathfrak{m}}$ la composante dans in d'un élément $X \in \mathrm{g}$ relative à la décomposition (4) de $\mathfrak{g}$. De même de $X_{\mathfrak{m}_{1}}$.

Remarquons que le groupe $B$ étant un sous-groupe de $L=L_{1} \times G_{2}$, on a ad. $x \cdot \mathfrak{w}_{1}=\mathfrak{w}_{1}$, ad. $x \cdot \mathfrak{n}_{1}=\mathfrak{n}_{1}$ et ad. $x \cdot g_{2}=g_{2}$ pour tout $x \in B$. Par suite, ad. $x \cdot \mathfrak{m}_{1}=m_{1}$ et ad. $x \cdot m=m$ pour tout $x \in B$. Maintenant la structure complexe invariante 
de $G / B$ définit un automorphisme $I$ de l'espace vectoriel m avec les propriétés suivantes :

1) $I^{2}=-1$;

2) ad. $x(I \cdot X)=I($ ad. $x \cdot X), x \in B, X \in m$ et en particulier $[Y, I \cdot X]=$ $I[Y, X], Y \in \mathfrak{b}, X \in \mathfrak{m}$

3) $I \cdot[X, Y]_{\mathfrak{m}}-[I \cdot X, Y]_{\mathfrak{m}}-[X, I \cdot Y]_{\mathfrak{m}}-I \cdot[I \cdot X, I \cdot Y]_{\mathfrak{m}}=0, X, Y \in \mathfrak{m}$.

Les variétés $L_{1} \cdot B / B$ et $G_{2} \cdot B / B$ étant des sous-variétés complexes de $G / B$, on a

4) $I \cdot \mathfrak{n}_{1}=\mathfrak{n}_{1}$;

5) $I \cdot g_{2}=g_{2}$.

Pour montrer que $G_{1} \cdot B / B$ est une sous-variété complexe de $G / B$, il suffit de montrer que $I \cdot \mathrm{m}_{1}=\mathrm{m}_{1}$. Soit $I \cdot X=I_{1} \cdot X+f(X)$ pour tout $X \in \mathrm{m}_{1}$, où $I_{1} \cdot X$ (resp. $f(X)$ ) désigne la composante de $I \cdot X \in m$ dans $m_{1}$ (resp. $g_{2}$ ) relative à la décomposition $m=m_{1}+g_{2}$. Alors $I_{1}$ est un endomorphisme de l'espace vectoriel $m_{1}$ et $f$ est une application linéaire de $m_{1}$ dans $g_{2}$. On va montrer que $f(X)=0$ pour tout $X \in m_{1}$. De 4) résulte que

4') $f(X)=0, X \in n_{1}$.

Par un calcul facile, on obtient de 1),2) et 3) les egalités suivantes:

1') $I_{1}^{2} \cdot X=-X, X \in \mathrm{m}_{1}$;

$\left.2^{\prime}\right)\left[Y, I_{1} \cdot X\right]=I_{1} \cdot[Y, X], Y \in \mathfrak{b}, X \in \mathrm{m}_{1}$;

3') $I_{1} \cdot[X, Y]_{\mathfrak{m}_{1}}-\left[I_{1} \cdot X, Y\right] \mathfrak{m}_{1}-\left[X, I_{1} \cdot Y\right] \mathfrak{m}_{1}-I_{1} \cdot\left[I_{1} \cdot X, I_{1} \cdot Y\right] \mathfrak{m}_{1}=0, X$, $Y \in m_{1}$;

$1^{\prime \prime)} f([Y, X])=0, Y \in \mathfrak{b}, X \in \mathrm{m}_{1}$;

$\left.2^{\prime \prime}\right) f\left([X, Y]_{\mathfrak{m}_{1}}\right)=f\left(\left[I_{1} \cdot X, I_{1} \cdot Y\right] \mathfrak{m}_{1}\right)+I[f(X), f(Y)], X, Y \in \mathfrak{m}_{1}$.

Les égalités $\left.1^{\prime}\right), 2^{\prime}$ ) et $\left.3^{\prime}\right)$ montrent que l'automorphisme $I_{1}$ de $m_{1}$ définit une structure complexe invariante de $G_{1} / B_{0}$.

Soit maintenant $\mathfrak{h}_{\mathfrak{b}}$ une sous-algèbre de Cartan de $\mathfrak{b}$ et soit $\mathfrak{h}_{\mathfrak{1}}=\mathfrak{n}_{\mathfrak{1}}+\mathfrak{h}_{\mathfrak{b}}$. Alors $\mathfrak{h}_{1}$ est une sous-algèbre de Cartan de $g_{1}$. Soit $g_{1}^{c}$ la complexification de $g_{1}$. Soit $D$ l'ensemble des racines non nulles de $g_{1}^{c}$ relatives à la sous-algèbre de Cartan $\mathfrak{h}_{1}^{c}$. Prolongeons $I_{1}$ en un automorphisme de l'espace vectoriel complexe $m_{1}^{c}$. Soit $m_{1}^{+}$(resp. $m_{1}^{-}$) le sous-espace de $m_{1}^{c}$ des éléments $X \in m_{1}^{c}$ tels que 
$I_{1} \cdot X=i \cdot X$ (resp. $\left.I_{1} \cdot X=-i \cdot X, i^{2}=-1\right)$. On a alors $m_{1}^{c}=m_{1}^{+}+m_{1}^{-}$. Soient $\mathfrak{n}_{1}^{+}=\mathfrak{n}_{1}^{c} \cap \mathfrak{m}_{1}^{+}$et $\mathfrak{n}_{1}^{-}=\mathfrak{n}_{1}^{c} \cap m_{1}^{-}$. De (4) résulte que $\mathfrak{n}_{1}^{c}=\mathfrak{n}_{1}^{+}+\mathfrak{n}_{1}^{-}$. Soit $D\left(\mathfrak{m}_{1}^{+}\right)$ (resp. $D\left(m_{1}^{-}\right)$) l'ensemble des racines $\alpha \in D$ telles que $E_{\alpha} \in \mathfrak{m}_{1}^{+}$(resp. $E_{\alpha} \in \mathfrak{m}_{1}^{-}$), où $E_{\alpha}$ désigne un élément de $g_{1}^{c}$ tel que $\left[H, E_{\alpha}\right]=\alpha(H) \cdot E_{\alpha}$ pour tout $H \in \mathfrak{l}_{1}^{c}$. Soit $\mathfrak{w}_{1}^{+}$(resp. $\mathfrak{w}_{1}^{-}$) le sous-espace vectoriel de $\mathfrak{m}_{1}^{+}$(resp. $\mathfrak{m}_{1}^{-}$) engendré linéairement par les $E_{\alpha}$ avec $\alpha \in \mathrm{D}\left(\mathfrak{m}_{1}^{+}\right)$(resp. $\alpha \in D\left(\mathfrak{m}_{1}^{-}\right)$). On a alors $\mathfrak{w}_{1}^{c}=\mathfrak{w}_{1}^{+}+\mathfrak{w}_{1}^{-}$ (voir [3]). Puisque $m_{1}^{c}=\mathfrak{n}_{1}^{c}+\mathfrak{w}_{1}^{c}$, on a $\mathfrak{m}_{1}^{c}=\mathfrak{n}_{1}^{+}+\mathfrak{w}_{1}^{+}+\mathfrak{n}_{1}^{-}+\mathfrak{m}_{1}^{-}, \quad m_{1}^{+}=\mathfrak{n}_{1}^{+}+\mathfrak{w}_{1}^{+}$, $m_{1}^{-}=\mathfrak{n}_{1}^{-}+\mathfrak{m}_{1}^{-}$. Prolongeons $f$ en une application linéaire complexe de $m_{1}^{c}$ dans $\mathrm{g}_{2}^{c}$. Montrons que $f(X)=0$ pour tout $X \in \mathrm{m}_{1}^{c}$. De $\left.4^{\prime}\right)$ résulte que $f(X)=0$ pour tout $X \in \mathfrak{n}_{1}^{c}$. Prenons un élément $E_{\alpha}$ avec $\alpha \in \mathrm{D}\left(\mathfrak{m}_{1}^{+}\right)$. Alors $E_{\alpha} \in \mathfrak{w}_{1}^{+}$. Soit $H \in \mathfrak{h}_{\mathfrak{b}}^{c}$. Alors il résulte de $\left.1^{\prime \prime}\right)$ que $f\left(\left[H, E_{\alpha}\right]\right)=0$. Or $f\left(\left[H, E_{\alpha}\right]\right)=\alpha(H)$ $f\left(E_{\alpha}\right)$ et par suite

$$
\alpha(H) f\left(E_{\alpha}\right)=0, \quad H \in \mathfrak{h}_{\mathfrak{b}}^{c},
$$

Soit maintenant $H \in \mathfrak{n}_{1}^{+}$. Alors $f(H)=0$ et de $\left.2^{\prime \prime}\right)$ résulte que $f\left(\left[H, E_{\alpha}\right]\right)=$ $f\left(\left[I_{1} \cdot H, I_{1} \cdot E_{\alpha}\right]\right)$. Puisque $H \in \mathfrak{n}_{1}^{+}$et $E_{\alpha} \in \mathfrak{w}_{1}^{+}$, on a $I_{1} \cdot H=i \cdot H$ et $I_{1} \cdot E_{\alpha}=i \cdot E_{\alpha}$. Par suite $f\left(\left[H, E_{\alpha}\right]\right)=-f\left(\left[H, E_{\alpha}\right]\right)$ et ceci entraîne $f\left(\left[H, E_{\alpha}\right]\right)=\alpha(H) f\left(E_{\alpha}\right)=0$. On a donc

$$
\alpha(H) f\left(E_{\alpha}\right)=0, \quad H \in \mathfrak{n}_{1}^{+} .
$$

De $(*)$ et $(* *)$ résulte que $\alpha(H) f\left(E_{\alpha}\right)=0$ pour tout $H \in \mathfrak{h}_{1}^{+}=\mathfrak{n}_{1}^{+}+\mathfrak{h}_{\mathfrak{b}}^{c}$. Or on sait que, si $\alpha \neq 0$, il existe un élément $H \in \mathfrak{h}_{1}^{+}$tel que $\alpha(H) \neq 0$ (voir [3], Lemma 7.1). On a alors $f\left(E_{\alpha}\right)=0$. Puisque $\mathfrak{r}_{1}^{+}$est engendré linéairement par $E_{\alpha}$, on a $f(X)=0$ pour tout $X \in \mathfrak{w}_{1}^{+}$. Par un raisonnement analogue on peut montrer que $f(X)=0$ pour tout $X \in \mathfrak{w}_{1}^{-}$. Par suite $f(X)=0$ pour tout $X \in \mathfrak{w}_{1}^{c}$. Il résulte de ce que on a démontre que $f(X)=0$ pour tout $X \in m_{1}^{c}$. Alors on a $I \cdot m_{1}=$ $\mathrm{m}_{1}$. La variété $G_{1} \cdot B / B$ est ainsi une sous-variété complexe de $G / B$.

On va montrer maintenant que la variété $G / L$ se munit d'une structure complexe invariante par $G$ telle que la projection canonique de $G / B$ sur $G / L$ soit holomorphe. On utilisera les notations introduites ci-dessus. L'automorphisme $I$ de $m$ laisse le sous-espace $\mathfrak{w}_{1}$ invariant. Soit $I^{\prime}$ la restriction de $I$ à $\mathfrak{w}_{1}$. On désignera par $X \mathfrak{w}_{1}$ la composante de $X \in g$ dans $\mathfrak{w}_{1}$ relative à la décomposition $g=\mathfrak{w}_{1}+\mathfrak{l}\left(\mathfrak{l}=l_{1}+g_{2}\right)$. On a alors les égalités suivantes :

a) $I^{2}=-1$ 
b) $\left[Y, I^{\prime} \cdot X\right]=I^{\prime} \cdot[Y, X], Y \in \mathfrak{l}, Y \in \mathfrak{w}_{1}$;

c) $I^{\prime} \cdot[X, Y]_{\mathfrak{w}_{1}}-\left[I^{\prime} \cdot X, Y\right]_{\mathfrak{w}_{1}}-\left[X, I^{\prime} \cdot Y\right]_{\mathfrak{w}_{1}}-I^{\prime} \cdot\left[I^{\prime} \cdot X, I^{\prime} \cdot Y\right]_{\mathfrak{w}_{1}}=0, X, Y \in$ $\mathfrak{w}_{1}$.

(cf. [3]). L'automorphisme $I^{\prime}$ de $\mathfrak{w}_{1}$ définit donc une structure complexe invariante de $G / L$. Il est facile de voir que la projection canonique de $G / B$ sur $G / L$ est holomorphe. Puisque $G=G_{1} \times G_{2}, \quad L=L_{1} \times G_{2}$, le groupe $G_{1}$ est transitif sur $W=G / L$ et le groupe d'isotropie de $G_{1}$ est égal à $L_{1}$. Le groupe $L_{1}$ étant le centralisateur d'un tore de $G_{1}, W$ est un espace homogène kählérien du groupe compact semi-simple $G_{1}$ (voir [2]).

On a ainsi démontré le théoréme suivant.

ThÉoRÈme $1^{\prime}$. Soit $V$ une variete complexe compacte et soit $G$ un groupe de Lie semi-simple connexe opérant sur $V$ de manière transitive, holomorphe et presque effective. Supposons que $V$ admette une mesure invariante par $G$. Soit $V=G / B$ et soit $B_{0}$ la composante connexe de l'elément neutre de B. Soient $G_{1}$ et $G_{2}$ la composante compacte et la composante ouverte de $G$. Alors $G_{2}$ est un groupe de Lie complexe. De plus, il existe un sous-groupe compact connexe $L_{1}$ du groupe compact $G_{1}$ avec les proprietés suivantes:

a) le groupe $L_{1}$ est le centralisateur d'un tore de $G_{1}$ dans $G_{1}$ et $B_{0}$ est un sous-groupe invariant de $L_{1}$ contenant la partie semi-simple de $L_{1}$. Le groupe $B_{0}$ est donc un C-sous-groupe de $G_{1}$ au sens de Wang;

b) soit $L=L_{1} \cdot G_{2}$. Alors le groupe $B$ est contenu dans $L$ et l'espace quotient $F=L / B$ est une sous-varieté compacte complexe parallélisable de $V=G / B$. Le groupe $L^{\prime}=L / B_{0}$ est un groupe complexe réductif et $F$ est un quotient de $L^{\prime}$ par un sous-groupe discret:

c) l'espace quotient $W=G / L$ est un espace homogène kählérien du groupe compact semi-simple $G_{1}$;

d) la variété $V$ est un espace fibré holomorphe de base $W$ et de fibre $F$.

5. Construction des varietés complexes compactes semi-simples. Soit $G_{1}$ un groupe de Lie compact semi-simple connexe et soit $G_{2}$ un groupe de Lie semi-simple complexe connexe et soit $G=G_{1} \times G_{2}$. Soit $L_{1}$ le sous-groupe de $G_{1}$ qui est le centralisateur d'un tore de $G_{1}$. Soit $B_{0}$ un sous-groupe fermé connexe de $L_{1}$ contenant la partie semi-simple de $L_{1}$ tel que $\operatorname{dim} L_{1}-\operatorname{dim} B_{0}$ soit pair. Alors $B_{0}$ est un sous-groupe invariant de $L_{1}$ et le groupe quotient $L_{1} / B_{0}$ 
est un tore. Prenons un sous-groupe discret $D$ du groupe $L^{\prime}=L_{1} / B_{0} \times G_{2}$ tel que l'espace quotient $L^{\prime} / D$ soit compact. Soit $B$ l'image inverse de $D$ dans $L=$ $L_{1} \times G_{2}$ par l'homomorphisme canonique de $L$ sur $L^{\prime}$. Alors $B$ est un sousgroupe fermé de $G$ contenu dans $L$ et l'espace quotient $G / B$ est compact. On voit facilement que le groupe $B$ est unimodulaire et donc que l'espace quotient $G / B$ admet une mesure invariante par $G$. On va montrer maintenant que l'espace $G / B$ admet une structure complexe invariante par $G$. Dans ce but on utilisera les notations introduites au paragraphe 4 . Soit donc

$$
\mathfrak{g}=m+\mathfrak{b}, \mathfrak{m}=m_{1}+\mathfrak{g}_{2}, m_{1}=\mathfrak{n}_{1}+\mathfrak{w}_{1}, \mathfrak{l}_{1}=\mathfrak{n}_{1}+\mathfrak{b}, \mathfrak{g}_{1}=\mathfrak{w}_{1}+\mathfrak{l}_{1}
$$

On sait qu'il existe une structure complexe invariante de l'espace quotient $G_{1} / B_{0}$ vérifiant les propriétés suivantes (coir [3] et [5]): Soit $I_{1}$ l'automorphisme de l'espace vectoriel $m_{1}$ defini par cette structure. Alors

1) $I_{1} \cdot \mathfrak{n}_{1}=n_{1}$;

2) $I_{1} \cdot \mathfrak{w}_{1}=\mathfrak{w}_{1}$ et la restriction $I^{\prime}$ de $I_{1}$ à $\mathfrak{w}_{1}$ définit une structure complexe invariante de $G_{1} / L_{1}$.

On voit facilement que ad. $x \cdot \mathfrak{n}_{1}=\mathfrak{n}_{1}$, ad. $x \cdot \mathfrak{w}_{1}=\mathfrak{w}_{1}$ et ad. $x \cdot g_{2}=g_{2}$ pour tout $x \in B$. On va montrer maintemant que ad. $x\left(I_{1} \cdot X\right)=I_{1}(\operatorname{ad} . x \cdot X)$ pour tout $x \in B$ et tout $X \in m_{1}$. Puisque $B$ est un sous-groupe de $L=L_{1} \times G_{2}$, tout élément $x$ de $B$ s'écrit $x=x_{1} \cdot x_{2}, x_{1} \in L_{1}, x_{2} \in G_{2}$. Pour tout $X \in m_{1}$ on a ad. $x \cdot X=$ ad. $x_{1} \cdot X$. Soit $X \in \mathfrak{n}_{1}$. L'espace vectoriel $\mathfrak{n}_{1}$ étant dans le centre de $l_{1}$, on a ad. $x_{1} \cdot X=X$ pour tout $X \in n_{1} . \quad$ Par suite ad. $x_{1}\left(I_{1} \cdot X\right)=I_{1} \cdot X$ et $I_{1}\left(\right.$ ad. $\left.x_{1} \cdot X\right)=$ $I_{1} \cdot X$ et donc ad. $x_{1}\left(I_{1} \cdot X\right)=I_{1}\left(\operatorname{ad} x_{1} \cdot X\right)$. Soit maintenant $X \in m_{1}$. Puisque la restriction de $I_{1}$ à $w_{1}$ définit une structure complexe invariante de $G_{1} / L_{1}$, on a ad. $y_{1}\left(I_{1} \cdot X\right)=I_{1}\left(\right.$ ad. $\left.y_{1} \cdot X\right)$ pour tout $y_{1} \in L_{1}$ et tout $X \in w_{1}$. En particulier ad. $x_{1}\left(I_{1} \cdot X\right)=I_{1}\left(\operatorname{ad} \cdot x_{1} \cdot X\right)$. Puisque $m_{1}=n_{1}+w_{1}$, il résulte de ce que on a montré que ad. $x(I \cdot X)=I($ ad. $x \cdot X)$ pour tout $x \in B$ et $X \in m_{1}$.

D'autre part, le groupe $G_{2}$ étant un groupe complexe, il existe un automorphisme $I_{2}$ de l'algèbre $g_{2}$ tel que $I_{2}^{2}=-1$ et que ad. $y\left(I_{2} \cdot X\right)=I_{2}(\operatorname{ad} . y \cdot X)$ pour tout $y \in G_{2}$ et tout $X \in g_{2}$. Soit maintenant $I$ un automorphisme de l'espace vectoriel $m=m_{1}+g_{2}$ défini par $I\left(X_{1}+X_{2}\right)=I_{1} \cdot X+I_{2} \cdot X_{2}, \quad X_{1} \in m_{1}, X_{2} \in g_{2}$. Il est facile de voir que $I$ définit une structure complexe invariante de $G / B$. L'espace quotient $G / B$ est ainsi une variété complex compacte semi-simple. Il résulte de ce que on a déjà montré que toute variété complexe compacte semi- 
simple se construit de cette manière.

6. Démonstration du theorème 2. Supposons maintenant que la variété $V=G / B$ soit kählérienne. Alors, la variété $F=L / B$ étant une sous-variété complexe de $V, F$ est aussi kählérienne. Or $F$ est parallélisable. D'après un théorème de Wang [6], $F$ est alors un tore complexe. Un groupe de Lie complexe semi-simple de dimension positive ne peut pas opérer sur un tore complexe de manière holomorphe et presque effective, car le plus grand groupe connexe d'automorphismes d'un tore complexe est abélien. Par suite on a $G_{2}=$ $(e)$ et donc $G=G_{1}$. Le groupe $G$ est ainsi compact. Alors $V$ admet une métrique kählérienne invariante par $G$ et par suite $V$ est un espace homogène kählérien du groupe compact semi-simple $G$. Le théorème 2 est ainsi démontré.

\section{BiBLIOGRAPHIE}

[1] A. Borel, Density properties for certain subgroups of semi-simple groups without compact components, Ann. of Math., 72 (1960), p. 179-188.

[2] A. Borel, Kählerian coset spaces of semi-simple Lie groups, Proc. Nat. Acad. U.S.A., 75 (1954), p. 1147-1151.

[3] J. Hano and S. Kobayashi, A fibering of a class of homogeneous complex manifolds, Trans. Amer. Math. Soc., 94 (1960), p. 233-243.

[4] Y. Matsushima, Sur les espaces homogènes kählériens d'un groupe de Lie réductif, Nagoya Math. Journ., 11 (1957), p. 53-60.

[5] H. C. Wang, Closed manifolds with homogeneous complex structure, Amer. Journ. Math., 76 (1954), p. 1-32.

[6] H. C. Wang, Complex parallisable manifolds, Proc. Amer. Math. Soc., 5 (1954), p. $771-$ 776.

Institut de Mathématiques

Université d'Osaka 